\title{
Experimental Study of Magnetic Slurries Regarding Magnetic Properties
}

\author{
G. Engdahl, Power Engineering, ABB Corporate Research, S-72178 Västerås, Sweden \\ B. Lerebourg, Ecole Nationale Supérieure d'Hydraulique et de Mécanique de Grenoble, F-38400 St Martin d'Hères, France
}

\begin{abstract}
The aim of this study was to assess the basic magnetic properties of a mixture composed of a commercial lubricant and soft magnetic iron particles, where the particle content is significantly higher than in conventional magnetic fluids, due to the fact that the particles verge on the sedimentation level. This so called magnetic slurry can, then, be regarded as a highly ductile magnetic conducting material for tentative use in magnetic circuit that can change shape under operation. The high particle content results in high saturation field densities that extend the application field towards electric power engineering. This paper, therefore, focuses on properties like relative permeability, losses, the $Q$-factor and the efficiency for the power frequencies 50 and $400 \mathrm{~Hz}$. It shows that the relative permeabilities are low (below 10). The values of the efficiency and the $Q$-factor decrease considerably above $0.5 \mathrm{~T}$. That limits the application of the studied slurry in electric power engineering. In such applications, the powder properties must be improved.
\end{abstract}

\section{INTRODUCTION}

The conventional magnetic fluids [1] have low magnetic saturation levels and can not, for that reason, be used in electric power engineering, for example in controllable reactive components. To make them usable in such applications, the particle density can be increased so that the mixture becomes a very ductile material with a considerably higher magnetic saturation level. This study, therefore, is devoted to the assessment of the magnetic properties of such mixtures.

\section{THE SLURRY}

\section{A. The Choice of the Slurry}

Powders for use in slurries in electric power engineering have to be made of a soft magnetic material, to be relatively non expensive, to be electrical non conducting and, finally, to have an ability to form dense and ductile slurries. Thus, two insulated iron powders from Höganäs $\mathrm{AB}$ were selected: the ABM.100.32 and the MHM.300.27 where the particle sizes are in the ranges $[30 \mu \mathrm{m} ; 200 \mu \mathrm{m}]$ and $[5 \mu \mathrm{m} ; 50 \mu \mathrm{m}]$, respectively. These powders are insulated which means that a thin iron oxide layer provides a very good internal electric insulation of the powder.

\section{B. The Choice of the Powder}

To get a homogeneous slurry, the powder has to be mixed with a more or less fluid matter, in this paper called "solvent." Its principal role is to enhance the particle motion and join the particles together forming a slurry rather than a dust cloud. Lubricants were, therefore, an appropriate choice regarding the "solvent." Different types of both commercial non expensive oils (mineral, synthetic and silicon oils) and mineral-oil-based greases have been tested regarding ductility and stability.

\section{The Preparation of the Mixture}

The results of the mixing tests showed that a homogeneous slurry with a particle density higher than 35 volume percents could not be obtained with any kind of grease. Furthermore, the mixing operation resulted in the entry of a huge amount of small air bubbles that could not be removed. This problem might be solved by heating the grease during the mixing.

With oils, the preparation procedure performed began by pouring the iron powder into a big amount of lubricant. The mixture was, then, carefully stirred to avoid air bubbles being trapped. After that, one let the mixture sedimentate and finally the oil surplus was removed. The particle density was, then, about 45 volume percents, independently of the type of oil. High-viscosity polyalphaolefine and silicon oils associated with the roughest powder (ABM.100.32) gave the best results regarding homogeneity and consistency of the slurry. The specification of the slurry whose experimental results are presented in this paper is the following: 54 volume percents of the polyalphaolefine oil MOBIL SHC $626\left(170 \mathrm{cSt}\right.$ at $\left.20^{\circ} \mathrm{C}\right)$ and 46 volume percents of the powder ABM.100.32 The density of the slurry was equal to $4.2 \mathrm{~g} / \mathrm{cm}^{3}$.

\section{The TeSTING EQUIPMENT}

The sketch of the testing equipment is represented in Fig. 1. It was composed of a $0.3 \mathrm{~mm}$-thick-strip wound core that has been truncated to make place for the sample, a cylindrical container of Plexiglass full of slurry, and an induction coil. Two $1 \mathrm{~mm}$-thick circular cliscs were placed on the top and the bottom of the container, respectively, to prevent the slurry from pouring out. These cliscs were made of a soft magnetic material, PERMEDYN [2], so as not to amount to an air gap regarding the magnetic field quantities. Finally, a centring device surrounded the sample with the aim of lining up the core and the sample and carrying the magnetic field intensity gauge.

A sinusoidal-voltage source supplied the induction coil. To prevent from big magnetic field distortions around the gap, a smaller sample (10 mm-high) also was designed. Thus, the general features of the testing equipment were:

Core cross-section: $32.1 \mathrm{~mm} \times 32.1 \mathrm{~mm}$

Container dimensions: Inner diameter: $32.1 \mathrm{~mm}$

Wall thickness: $0.1 \mathrm{~mm}$

Height: $48 \mathrm{~mm}$ for $\mathrm{B}<0.6 \mathrm{~T}$

$10 \mathrm{~mm}$ for $0.6 \mathrm{~T}<\mathrm{B}<1 \mathrm{~T}$ 


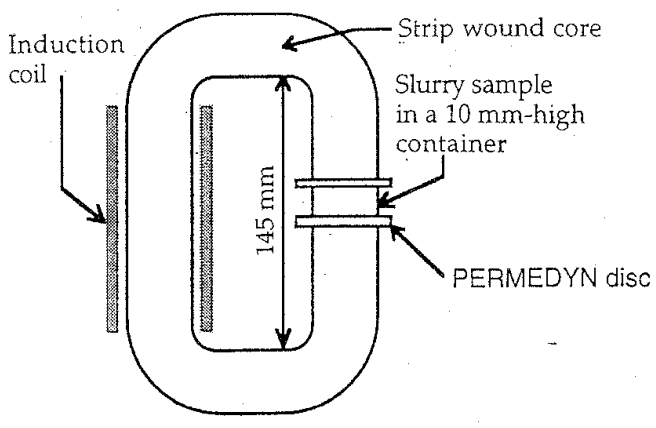

Fig. 1. The testing equipment

\section{THE MEASUREMENT EQUIPMENT}

\section{A. The Magnetic Field Quantity Gauges}

To measure the magnetic field intensity inside the sample a Rogowsky coil was placed beside the sample, see Fig. 2. The number of turns of the coil, N, was approximately 1800 .

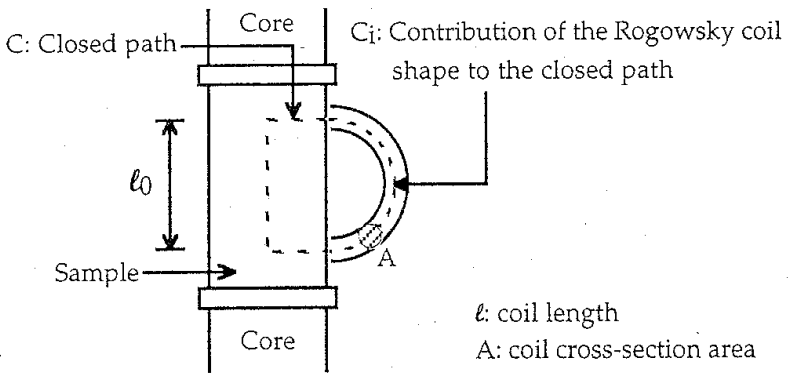

Fig. 2. Position of the Rogowsky coil beside the sample

The relationship between the voltage, e, at the Rogowsky coil terminals and the magnetic field intensity inside the sample, $\mathrm{H}_{\mathrm{int}}$, was calculated by means of the Equation (1).

$$
\int_{C} \overrightarrow{\mathrm{H}} \overrightarrow{\mathrm{d} \ell}=\mathrm{NI}=0
$$

Knowing that $\mathrm{H}=\mathrm{B} / \mu_{0}$ outside the sample and, therefore, in the coil, the Lenz's law gives the relationship between the contribution of the coil to this integral and the voltage at its terminals. Finally, one get:

$$
H_{\text {int }}=\frac{\ell}{\ell_{0}} \frac{1}{N \mu_{0} \mathrm{~A}} \int e d t=K_{1} \int e d t
$$

B was measured by means of a very thin coil with 8 turns that surrounded the sample at his half height.

\section{B. The Recording of the Signals}

To get the B-H hysteresis loop, the magnetic field quantities were simultaneously recorded at several instants in a transient recorder, during at least one period, $\mathrm{T}$.

\section{THE DATA PROCESSING}

The recorded signals were, then, processed in order to obtain the hysteresis loops, the relative permeabilities, the magnetic losses, etc.

\section{A. The Relative Permeability Computation}

For each test, $\mu_{r}$ was estimated as the minimal value of the differential relative permeability function $1 / \mu_{0} \mathrm{~dB} / \mathrm{dH}$, that is, graphically, the slope of the tangent of the B-H loop at its peak points.

\section{B. The MagneticLosses}

In Fig. 3, a typical anomaly of a hysteresis loop caused by the lack of measurement precision is shown. That appeared especially at high induction.

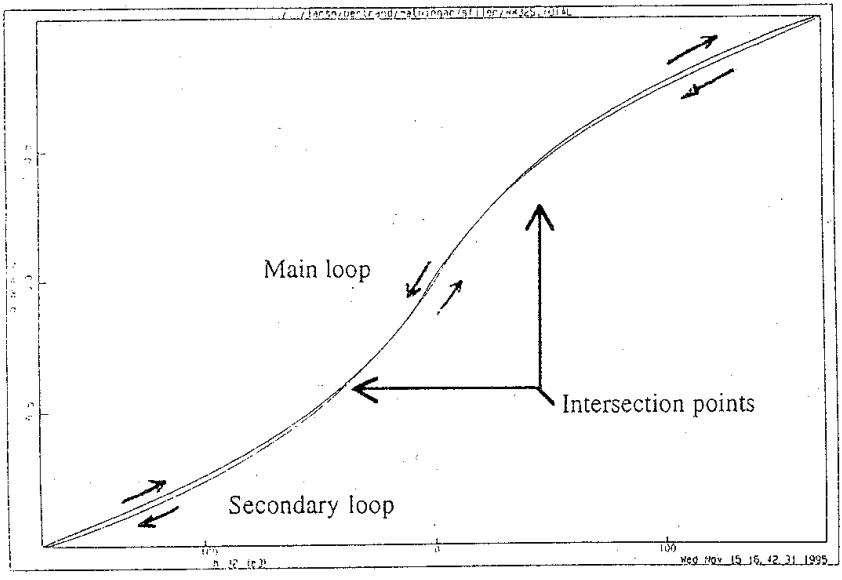

Fig. 3. Example of a loop with two intersection points

The intersection points of the loops appeared in the neighborhood ( $\mathrm{H}= \pm 16,000 \mathrm{~A} / \mathrm{m} ; \mathrm{B}= \pm 0.3 \mathrm{~T}$ ), independently of the magnetic field intensity top-value during the tests. As a consequence, the higher the magnetization, the bigger the secondary loops compared to the main loop. Therefore, the magnetic losses were computed as the absolute summation of all the areas, though this computation may have overestimated them.

\section{The Q-Factor and Efficiency Calculations}

The Q-factor represents the number of periods necessary to dissipate by losses the maximum reactive energy in the sample:

$$
Q=\frac{U_{\text {top }}}{\text { Losses under one period }}=\frac{U_{\text {top }}}{\int_{\mathrm{T}}^{\mathrm{BdH}}}
$$

To compute it, the reactive volume energy, $\mathrm{U}$, has been approximated. In our case, $\delta \mathrm{U}=\mathrm{H} \delta \mathrm{B}=\mathrm{H} \partial \mathrm{B} / \partial \mathrm{H} \delta \mathrm{H}$. Then, by neglecting the second order term of the mathematical expansion $\delta\left(1 / 2 \mathrm{H}^{2} \partial \mathrm{B} / \partial \mathrm{H}\right)=\mathrm{H} \partial \mathrm{B} / \partial \mathrm{H} \delta \mathrm{H}+1 / 2 \mathrm{H}^{2} \delta(\partial \mathrm{B} / \partial \mathrm{H})$, one get:

$$
\mathrm{U}=1 / 2 \mathrm{H}^{2} \partial \mathrm{B} / \partial \mathrm{H}
$$

The efficiency, $\eta$, compares the magnetic power losses to the reactive power: 


$$
\eta=1-\frac{\frac{1}{\rho \mathrm{T}} \int_{\mathrm{T}} \mathrm{BdH}}{\frac{1}{\rho \mathrm{T}} \int_{\mathrm{T}}\left|\frac{\partial \mathrm{U}}{\partial \mathrm{t}}\right| \mathrm{dt}}
$$

\section{THE RESULTS}

The computed minimal relative permeabilities were low, decreased slowly by the magnetic field density, and did not depend on the frequency:

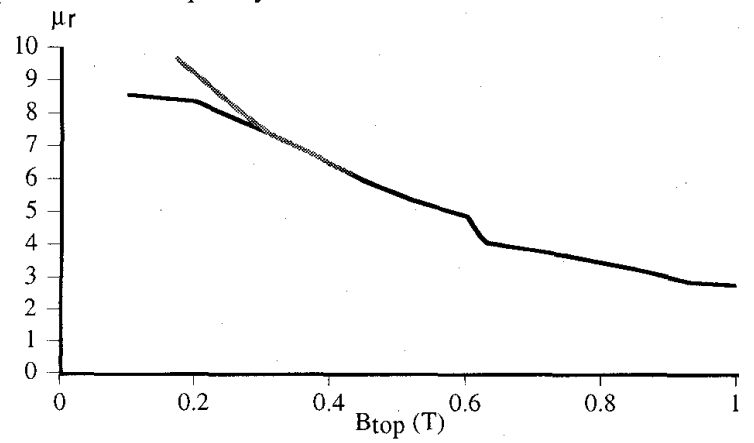

Fig. 4. Relative permeabilities at $50 \mathrm{~Hz}$ (black line) and $400 \mathrm{~Hz}$ (gray line)

The difference between the two curves can be ascribed to the imprecision of the graphic estimation of the slope at $0.17 \mathrm{~T}$ at $400 \mathrm{~Hz}$. The corresponding magnetization curve, see in Fig. 5, also showed the same shape for both frequencies:

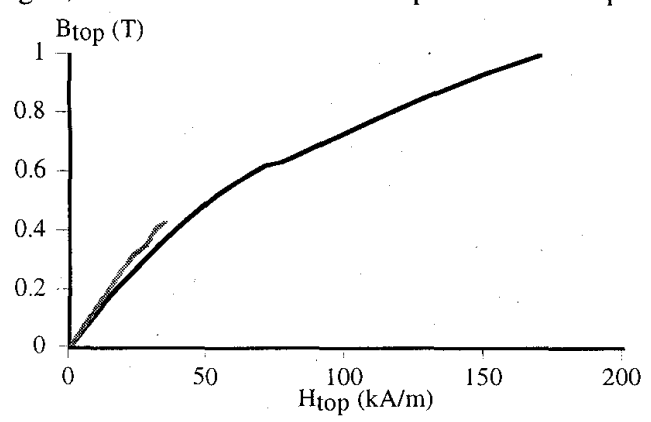

Fig. 5. Relation between $B_{\text {top }}$ (peak value of magnetic field density) and $\mathrm{H}_{\text {top }}$ at $50 \mathrm{~Hz}$ (black line) and $400 \mathrm{~Hz}$ (gray line)

In Fig. 6 and Fig. 7, the magnetic losses are compared with the soft magnetic material PERMEDYN.

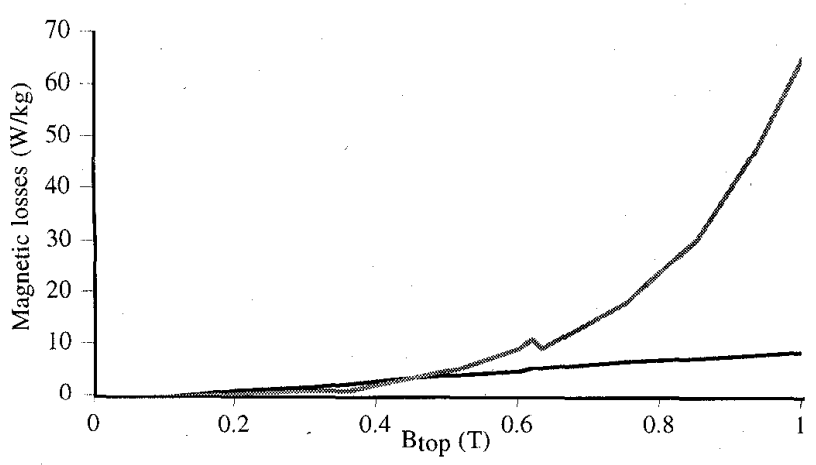

Fig. 6. Losses comparison at $50 \mathrm{~Hz}$ between PERMEDYN (black line) and the studied slurry (gray line)
The drop observed near $0.6 \mathrm{~T}$ corresponds to the mentioned sample change. Just below $0.6 \mathrm{~T}$, the magnetic field intensity was probably distorted resulting in an overestimation of the losses. Despite that, the losses of the studied slurry are considered too high compared to the PERMEDYN which is made of a similar iron powder from the same supplier, Höganäs.

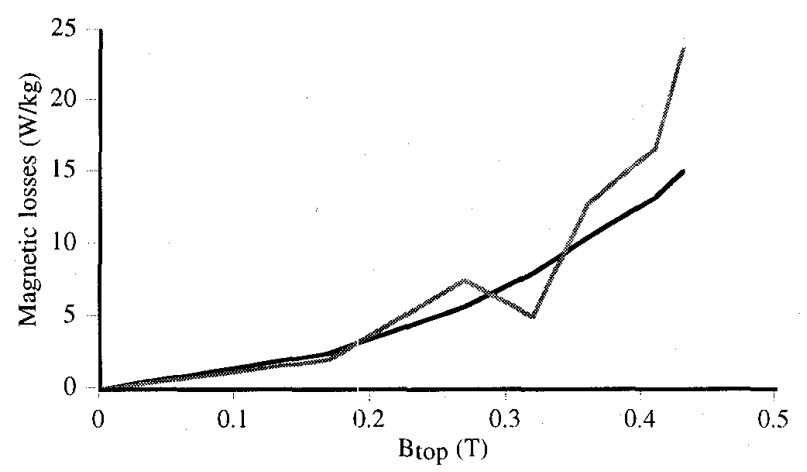

Fig. 7. Losses comparison at $400 \mathrm{~Hz}$ between PERMEDYN (black line) and the studied slurry (gray line)

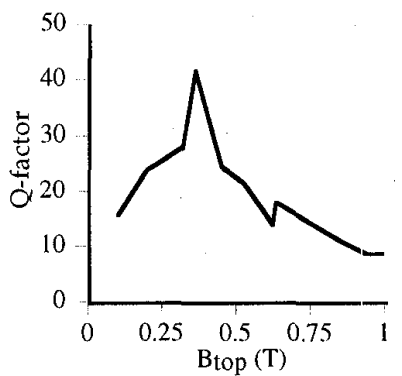

Fig. 8. (a) Q-factor at $50 \mathrm{~Hz}$

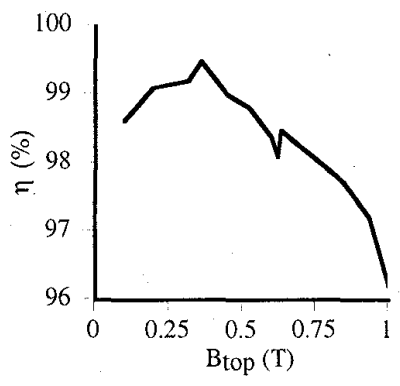

(b) Efficiency at $50 \mathrm{~Hz}$
The sudden jump of $Q$ and $\eta$ around $0.6 \mathrm{~T}$ in the Fig. 8(a) and 8 (b) can be explained in the same way as the drop of the magnetic losses in the Fig. 4. Thus, the Q-factor and the efficiency below $0.6 \mathrm{~T}$ may be a little higher than these shown.

\section{CONCLUSIONS}

The optimal use of the studied slurry is in the neighborhood of $\mathrm{B}_{\text {top }}=0.4 \mathrm{~T}$. This value is considerably higher than those in conventional magnetic fluids, which indicates that use of magnetic slurries can be feasible in electric power applications. However, the powder quality, regarding hysteresis. losses at higher field densities, must be improved.

\section{REFERENCES}

[1] B. D. Moskowitz and K. Raj, Ferrofluidics Corporation, Nashua, NH, USA, personal communication, 1995

[2] T. Cedell, "Magnetostrictive materials and selected applications," doctoral thesis, Lund University, Sweden, 1995, pp. 165-188. 